GLOBAL JOURNAL OF ENGINEERING RESEARCH VOL 12, 2013: 1-12

\title{
FDTD MODELLING OF ELECTROMAGNETIC WAVES IN STRATIFIED MEDIUM
}

N. FARUK AND U. M. GANA

(Received 15, October 2012; Revision Accepted 29, April 2013)

\begin{abstract}
A time domain numerical procedure is presented for a simulation of electromagnetic wave phenomena. The technique is an adaptation of the finite-difference time domain (FDTD) approach usually applied to model electromagnetic wave propagation. In this paper a simple 2D implementation of FDTD algorithm in mathematica environment is presented. Source implementation and the effect of conductivity on the incident field are investigated. Simple illustrations of propagation in a non-conducting, partial conducting and conducting medium are provided. For the computational space, Cartesian grids of fixed size were used as it makes grid generation to be relatively easy. The numerical data generated by the program code were sampled at various time steps from $t 0=1$ to 90 along the computational space. The simulation results show the advancement of the pulse into the medium at various time stepping, shift in the peak of the amplitude was observed on the pulse for all the time steps. An attempt to further show the attenuation as the wave propagates into the stratified medium is made. The amplitude of the pulse falls sharply from 0.006 to $1 \times 10^{-11}$ for $\mathrm{t} 0=1$ and $\mathrm{t} 0=50$. The results indicate the working of the model and it could be used to study the behavior of the wave as it does propagate across the medium.
\end{abstract}

KEYWORDS: Stratified Medium, Finite Difference Time Domain (FDTD), mathematica, Maxwell's Equations, Electromagnetic Waves (EM)

\section{INTRODUCTION}

Maxwell's equations provide a description of electromagnetic phenomenon often mathematical difficulties are usually encountered while trying to solve the equations in the circumstances of practical applications. In a few special cases, it is possible to obtain exact analytic or approximate solutions. However in most cases of practical interest, numerical techniques are applied to obtain the approximate solutions. The finite difference time domain (FDTD) method [1] is a full-wave and powerful numerical method for solving Maxwell's equations. The technique is one of the key simulation tools in study of electromagnetic propagation [2]. It is also a popular electromagnetic modelling technique in the general class of differential time domain numerical modelling methods. Other methods used today, include moment and element method. An important feature of FDTD method is that, the wave equation which is second order partial differential equations has to be expressed as two coupled first order partial differential equations. The method is an initial value problem formulation, thus the solution at any future time depend explicitly on the solution at earlier times [1][3]. When Maxwell's differential form equations are examined, it can be seen that the time derivative of the electric field ( $E$ field) is dependent on the curl of the magnetic field $(\mathrm{H}$ field). This can be simplified to state that the change in the $E$ field (time derivative) is dependent on the change in the $\mathrm{H}$ field across space (the curl); these result in the FDTD equation.

Although all electromagnetic phenomena

N. Faruk, Department of Telecommunication Science, University of Ilorin, Ilorin, Nigeria.

U. M. Gana, Department of Physics, Bayero University, Kano, Nigeria. 
can be studied in empty space, this is an important part of any introductory study of wave propagation. In this paper, we embark upon a study of the electromagnetic waves in stratified medium. The theory of stratified media is concerned with the propagation of linearly polarized harmonic waves in systems of media which are either part wise homogeneous and/or normally inhomogeneous, i.e. their optical constant depends on the depth measured along the normal to the layer [4]. By stratified medium means medium that their index of refraction is a function of variable. This variable could be conductivity, permeability or permittivity of the medium. The propagation of EM waves in space is important from practical point of view. Astronomical information concerning particles in space their nature, distribution and shapes are obtainable from such studies. In metrology and mineralogy the study also allows deductions about climatic changes and for the forecast/prediction of weather changes. The study has gained a great deal of importance because of technological applications and developments.

\section{Related Works}

In an attempt to solve the Maxwell's equations for practical relevance, several papers have been published. [5], presents 1D FDTD model for Maxwell's equation and subsequent implementation using MATLAB. The paper successfully demonstrates a working 1D-FDTD code that correctly implements PEC (perfect electric conductors) and PMC (Perfect magnetic conductors) boundaries. The code was also applied to investigate pulse reflections from dielectric slabs of various thicknesses and it was also found through simulation that, increasing the thickness of the slabs drastically reduces the lowreflectivity bandwidth. Similarly, Ikata [3], gives the numeral solution of the Maxwell's curl relation using central difference equation, in the work, FORTRAN code were used for the implementation of the algorithm by propagating half-sine wave into three different medium i.e. non conducting, partial conducting and conducting medium. Also, [6] presents two methods for calculating the reflection of EM plane wave from a stratified media, backed by a PEMC (perfect electromagnetic conductors) based on FDTD technique. The first is an analytic approach, based on the propagators and wavesplitting technique and the second is the numerical FDTD method. Hybrid finite element finite-difference time-domain (FE/FDTD) technique for solving complex electromagnetic problems was presented in [7], this method combines the computational simplicity of the structured FDTD scheme with the flexibility of the finite-element method (FEM) thus, these enables them to accurately model curved geometries, numerical results from their paper illustrates the accuracy of the method. [8], also present FDTD modeling of scatters in stratified media, the paper discusses a method whereby the electromagnetic fields scattered by a buried object can easily be calculated by the FDTD technique. One of the advantages of FDTD techniques is its simplicity and straightforward to implement as compared with FE method, also, the use of Cartesian grids makes grid generation to be relatively easy. In terms of memory requirement, the technique has proven most effective. One of the drawbacks of this technique is inability to model curve geometrical objects, as grids would usually provide approximations of the geometry of the structure with little details. Despite the limitations of this technique, it is still found useful in various real life applications.

The FDTD modelling studies has been proven highly significant and has been applied to model and solve real life application problems. FDTD models have been applied extensively in the field of geoscience. Muhkopadhyay et al [9], present an implementation of parallel 3D FDTD technique for propagating radar EM waves in borehole, the simulation study demonstrates that, borehole radar can be useful for mining as well as oilfield applications for imaging electrical discontinuity with high resolution. The model was also applied for case of layered medium and was found that, the amplitude of the propagating wave varies with the thickness of the sand layer. This property can be utilized to estimate the dielectric permittivity of the medium. The effect of borehole on radar traces has also been noticed; the radar traces are affected by the presence of borehole, layer thickness and scattering from sharp edges. The shape, signature and the initial travel time of the received waveform changes as the wave does propagates into the starter. Similar studies have been presented in [10] [11] and [12]. Therefore, it would be of great importance and relevance to develop a model for FDTD for practical relevance.

This work is a modification to that presented in [3], the major enhancement is the methodology in solving the equations and 
implementation. In this paper, we present a solution of Maxwell's curl relation using forward difference method; codes were generated and subsequently, implementation in the mathematica environment version 7.0. The paper is organized as follows: Section II, provides related work, FDTD formulation is presented in section III; Section IV provides the simulation results and finally, Section $\mathrm{V}$ concludes the paper.

\section{FDTD FORMULATION}

A. SOLUTION OF MAXWELL'S CURL DIFFERENTIAL EQUATION USING FDTD METHOD

Consider symmetric form of Maxwell's curl relation in source free linear medium.

$\operatorname{curl} H=\varepsilon \frac{\partial E}{\partial t}+\sigma E$

curlE $=-\mu \frac{\partial H}{\partial t}-\sigma_{m} E$

Where $\sigma_{m}$ an equivalent magnetic conductivity, and the medium parameters are assumed to be time independent.

From (2.0) $\nabla \times E=-\mu \frac{\partial H}{\partial t}-\sigma_{m} H$

$$
\begin{array}{r}
\frac{\partial H_{x}}{\partial t}=-\frac{1}{\mu}\left\{\left(\frac{\partial E_{z}}{\partial y}-\frac{\partial E_{y}}{\partial z}\right)+\sigma_{m} H_{x}\right\} \\
\text { Then, } \frac{\partial H_{y}}{\partial t}=\frac{1}{\mu}\left\{\left(\frac{\partial E_{z}}{\partial x}-\frac{\partial E_{x}}{\partial z}\right)-\sigma_{m} H_{y}\right\}
\end{array}
$$

$\frac{\partial H_{z}}{\partial t}=\left\{\left(\frac{\partial E_{x}}{\partial y}-\frac{\partial E_{y}}{\partial x}\right)-\sigma_{m} H_{z}\right\}$

similarly

$$
\begin{aligned}
& \frac{\partial E_{x}}{\partial t}=\frac{1}{\varepsilon}\left(\frac{\partial H_{z}}{\partial y}-\frac{\partial H_{y}}{\partial z}-\sigma E_{x}\right) \\
& \frac{\partial E_{y}}{\partial t}=\frac{1}{\varepsilon}\left(\frac{\partial H_{x}}{\partial z}-\frac{\partial H_{z}}{\partial x}-\sigma E_{y}\right) \\
& \frac{\partial E_{z}}{\partial t}=\frac{1}{\varepsilon}\left(\frac{\partial H_{z}}{\partial x}-\frac{\partial H_{x}}{\partial y}-\sigma E_{z}\right) .
\end{aligned}
$$

A discretization scheme for equation (3.0) consists in using a Taylor series expansion for a function $f(x)$ and writing the first derivative of $f(x)$ as:

$\frac{\partial f\left(x_{0}\right)}{\partial x}=\frac{f\left(x_{0}+\Delta / 2\right)-f\left(x_{0}-\Delta / 2\right)+o\left(\Delta^{2}\right)}{\Delta}$

Where $x_{0}$ is the point at which the derivative is evaluated and $\Delta$ is a small increment and it is usually to denoted space grid points;

$$
(i, j, k)=(i \Delta x, j \Delta y, k \Delta z)
$$

And any function of space and time by; 
$f^{n}(i, j, k)=f(i \Delta x, j \Delta y, k \Delta z)$

Where $i, j$ and $k$ are integers which locate a grid points, $\Delta x, \Delta y, \Delta z$ are space increments along the respective axes and assumed to be same size, $\Delta t$ is a time increment and $n$ is an integer denoting the number of time steps.

Applying the discretization procedure (5.0) to (3.0) leads to their forward finite difference equivalent, in a homogeneous medium.

$$
\begin{aligned}
& H_{x}^{n+1}(i, j+1, k+1)-H_{x}^{n}(i, j, k)=\frac{\Delta t}{\Delta \mu}\left\{E_{y}(i, j, k+1)-E_{y}(i, j, k)+E_{z}(i, j, k)-E_{z}(i, j+1, k)-\sigma_{m} H_{x}(i, j, k)\right\} \\
& H_{x}^{n+1}(i, j+1, k+1)=\left(1-\frac{\Delta t \sigma_{m}}{\mu}\right) H_{x}^{n}(i, j, k)+ \\
& \frac{\Delta t}{\Delta \mu}\left\{E_{y}(i, j, k+1)-E_{y}(i, j, k)+E_{z}(i, j, k)-E_{z}(i, j, k+1)\right\} \ldots \ldots \ldots \ldots \ldots \ldots . . .(8)
\end{aligned}
$$

By considering $\frac{\Delta t \sigma_{m}}{\mu}<<1$, the corresponding FDTD equation (3.0) in $y$ and $z$ coordinate

$$
\begin{aligned}
& H_{y}^{n+1}(i+1, j, k+1)=\left[\frac{1-\sigma_{m} \Delta t / 2 \mu}{1+\sigma_{m} \Delta t / 2 \mu}\right] H_{y}^{n}(i, j, k)+ \\
& \frac{\Delta t}{\Delta \mu}\left[E_{z}^{n}(i+1, j, k)-E_{z}^{n}(i, j, k)+E_{x}^{n}(i . j . k)-E_{x}^{n}(i . j . k+1)\right] \ldots \ldots . \\
& H_{z}^{n}(i+1, j+1, k)=\left[\frac{1-\sigma_{m} \Delta t / 2 \mu}{1+\sigma_{m} \Delta t / 2 \mu}\right] H_{z}^{n}(i, j, k)+ \\
& \frac{\Delta t}{\Delta \mu}\left\{E_{x}^{n}(i, j+1, k)-E_{x}^{n}(i, j, k)+E_{y}^{n}(i, j, k)-E_{y}^{n}(i+1, j, k)\right\} .
\end{aligned}
$$

Similarly for the electric field vector $E$, we have:

$$
\begin{aligned}
& E_{x}^{n+1}(i+1, j, k)=\left[\frac{1-\sigma_{m} \Delta t / 2 \varepsilon}{1+\sigma_{m} \Delta t / 2 \varepsilon}\right] E_{x}^{n}(i, j, k)+ \\
& \frac{\Delta t}{\Delta \varepsilon}\left\{H_{z}^{n+1}(i+1, j+1, k)-H_{z}^{n}(i, j, k)+H_{y}^{n}(i, j, k)-H_{y}^{n+1}(i+1, j, k+1)\right\} .
\end{aligned}
$$




$$
\begin{aligned}
& E_{y}^{n+1}(i, j+1, k)=\left[\frac{1-\sigma_{m} \Delta t / 2 \varepsilon}{1+\sigma_{m} \Delta t / 2 \varepsilon}\right] E_{y}^{n}(i, j, k)+ \\
& \frac{\Delta t}{\Delta \varepsilon}\left\{H_{x}^{n+1}(i, j+1, k+1)-H_{x}^{n}(i, j, k)+H_{z}^{n}(i, j, k)-H_{z}^{n+1}(i+1, j+1, k)\right\} . \\
& E_{z}^{n+1}(i, j, k+1)=\left[\frac{1-\sigma_{m} \Delta t / 2 \varepsilon}{1+\sigma_{m} \Delta t / 2 \varepsilon}\right] E_{z}^{n}(i, j, k)+ \\
& \frac{\Delta t}{\Delta \varepsilon}\left[H_{y}^{n+1}(i+1, j, k+1)-H_{y}^{n}(i, j, k)+H_{x}^{n}(i, j, k)-H_{x}^{n+1}(i, j+1, k+1)\right] .
\end{aligned}
$$

Eqn (9) is used to compute new value for a field component at an interior grid point, and it depends on its preceding value of the components of the other vector at adjacent points. The FDTD algorithm (8-13) is a direct point-by-point approximation of the derivatives in the time independent Maxwell curl relations. Using forward difference this interpretation is useful in understanding how FDTD models wave propagations.

\section{REDUCTION OF MAXWELL'S EQUATIONS TO 2D MODEL}

For computational complexity and the memory requirement for the FDTD simulation; it is common to find FDTD simulation experiments implemented on a 2-D lattice. In this paper, we assume that the field components don't depend on the $z$ coordinate $(\partial / \partial z=0)$. In this regards the plane electromagnetic field can be decomposed into transverse electric (TE) and transverse magnetic (TM) waves. These two waves are described by the relations:

1) TE: $E_{z}=0: H_{x}=H_{y}=0$

2) TM: $H_{z}=0 ; E_{x}=E_{y}=0$

Considering TM wave then the corresponding finite difference relations are:

$$
\begin{aligned}
& E_{z}^{n+1}(i, j)=\left[\frac{1-\sigma \Delta t / 2 \varepsilon}{1+\sigma \Delta t / 2 \varepsilon}\right] E_{z}{ }^{n}(i, j)+\frac{\Delta t / \varepsilon \Delta}{1+\sigma \Delta t / 2 \varepsilon} \\
& {\left[H_{y}{ }^{n+1}(i+1, j)-H_{y}{ }^{n+1}(i-1, j)+H_{x}{ }^{n+1}(i, j-1)-H_{x}{ }^{n+1}(i, j+1)\right]} \\
& H_{x}{ }^{n+1}(i, j+1)=\left[\frac{1-\sigma_{m} \Delta t / 2 \mu}{1+\sigma_{m} \Delta t / 2 \mu}\right] H_{x}{ }^{n-1}(i, j+1)+\frac{\Delta t / \mu \Delta}{1+\sigma_{m} \Delta t / 2 \mu}\left[E_{z}{ }^{n}(i, j)-E_{z}{ }^{n}(i, j+1)\right] \ldots \ldots . . \\
& H_{y}{ }^{n+1}(i+1, j)=\left[\frac{1-\sigma_{m} \Delta t / 2 \mu}{1+\sigma_{m} \Delta t / 2 \mu}\right] H_{y}{ }^{n-1}(i+1, j)+\frac{\Delta t / \mu \Delta}{1+\sigma_{m} \Delta t / 2 \mu}\left[E_{z}{ }^{n}(i+1, j)-E_{z}{ }^{n}(i, j)\right] \ldots
\end{aligned}
$$




\section{SIMULATION RESULTS}

\section{A. INITIALIZATION}

The evaluation of the set of equations in section $\mathrm{V}$, on introducing the initial values $E_{z}^{n}(i, j)$ at the starting time $\mathrm{t}=0$. Initializing the algorithm by putting $\mathrm{n}=0$ in (15) and (16) gives

$$
\begin{aligned}
& H^{1}{ }_{x}(i, j+1)=\frac{\Delta t}{\mu \Delta}\left[E^{0}{ }_{z}(i, j)-E^{0}{ }_{z}(i, j+1)\right] . \\
& H^{1}{ }_{y}(i+1, j)=\frac{\Delta t}{\mu \Delta}\left[E^{0}{ }_{z}(i+1, j)-E_{z}^{0}(i, j)\right] .
\end{aligned}
$$

\section{B. ALGORITHM}

This section describes the algorithm for the stratified medium. Wolfram Mathematica environment version 7.0 was used for implementation of the algorithm. Wolfram mathematica is founded by Stephen Wolfram in 1987 [13]. Wolfram Research is one of the world's most respected software companies--as well as a powerhouse of scientific and technical innovation. Mathematica is a computational software program used in scientific, engineering, and mathematical fields and other areas of technical computing. It was conceived by Stephen Wolfram and is developed by Wolfram Research of Champaign, Illinois [14]. It has wide range of tools for complex number, arbitrary precision, interval arithmetic, symbolic computation, 2D and 3D data and function visualization and many more. Mathematica has capabilities for connecting to DLLs. SQL, Java, .NET, C++, FORTRAN, CUDA and MATLAB

Algorithm: In this subsection the pseudo codes which implement the finite difference time domain algorithm to produce the results described is given. See Appendix A for nomenclature.

1: Generating Pulse (The electric field in the z-direction)

gaussian[x_, $\left.\mathrm{y}_{-}\right]:=\operatorname{Exp}\left[-\left((\mathrm{x}-4)^{\wedge} 2+(\mathrm{y}-5)^{\wedge} 2\right) / 10\right]$;

pulse $\left[x_{-}, y_{-}\right]:=$gaussian $[x, y]$;

If $x>x \mid$ And $x<x u$ Then

If $y>y l$ And $y<y u$ Then

$E z(i, j):=$ gaussian $[x, y]$;

End If

Else

End If

$E z(i, j)=0 \#$

2. Initializing array $\mathrm{Hx} \& \mathrm{Hy}$ (Magnetic fields in the $\mathrm{x}$ and $\mathrm{y}$-direction)

For $\mathrm{i}=1$ To $\mathrm{Nx}$

For $\mathrm{j}=1$ To Ny

$\mathrm{Hxt}(\mathrm{i}, \mathrm{j})=-\mathrm{DelT}^{*}(\operatorname{Ezt}(\mathrm{i}, \mathrm{j})-\operatorname{Ezt}(\mathrm{i}, \mathrm{j}-1)) /$ muy

$\operatorname{Hyt}(\mathrm{i}, \mathrm{j})=\operatorname{DelT}^{*}(\operatorname{Ezt}(\mathrm{i}, \mathrm{j})-\operatorname{Ezt}(\mathrm{i}-1, \mathrm{j})) / \operatorname{mux}$

3. Initializing Time Step (Time stepping loop)

For $\mathrm{i}=\mathrm{N} \times 1$ To $\mathrm{N} \times 2-1$

$$
\begin{aligned}
& \text { For } \mathrm{j}=\mathrm{Ny} 1+1 \mathrm{To} \mathrm{Ny} 2-1 \\
& \text { Ezt(i, j + Ny2 })=E z(i, j+N y 2)+\operatorname{DelT} *(H y t(i+1, j+N y 2)-H y t(i, j+N y 2)) / e p x \\
& - \text { DelT * }(H x t(i, j+N y 2+1)-H x t(i, j+N y 2)) / \text { epy } \\
& \mathrm{T}=\mathrm{T}+\mathrm{DelT}
\end{aligned}
$$


4. Advancing $\mathbf{H x} \& \mathrm{Hy}$ (Advancing the Magnetic fields in the $\mathrm{x}$ and $\mathrm{y}$-direction)

For $\mathrm{i}=\mathrm{N} x 1+1$ To $\mathrm{N} x 2-1$

For $\mathrm{j}=\mathrm{Ny} 1+1$ To Ny2 -1

$H x t(i, j+N y 2)=H x t(i, j+N y 2)-\operatorname{DelT}{ }^{*}(E z t(i, j+N y 2)-E z t(i, j+N y 2-1)) /$ muy

Hyt $(i, j+N y 2)=H y t(i, j+N y 2)+\operatorname{DelT}{ }^{*}(E z t(i-1, j+N y 2)-E z t(i, j+N y 2)) /$ mux

5. Set Correction (setting corrections and rewriting the Electric Field component in z-direction)

For $\mathrm{i}=1$ To $\mathrm{Nx}$

$\operatorname{Ezt}(i, 0)=\operatorname{Ezt}(i, 1)$

Ezt(i, Ny) $=$ Ezt $(\mathrm{i}, \mathrm{Ny}-1)$

For $\mathrm{j}=1$ To $\mathrm{Ny}$

$\operatorname{Ezt}(0, \mathrm{j})=\operatorname{Ez}(1, \mathrm{j})+\operatorname{rCLF}^{*}(\operatorname{Ez}(0, \mathrm{j})-\operatorname{Ezt}(1, \mathrm{j}))$

$\operatorname{Ezt}(N x, j)=E z(N x-1, j)+r C L F{ }^{*}(E z(N x-1, j)-E z t(N x-1, j))$

$\operatorname{Ezt}(0,0)=\operatorname{Ezt}(0,1)$

$\operatorname{Ezt}(0, \mathrm{Ny})=\operatorname{Ezt}(0, \mathrm{Ny}-1)$

$\operatorname{Ezt}(N x, 0)=\operatorname{Ezt}(N x, 1)$

$\operatorname{Ezt}(\mathrm{Nx}, \mathrm{Ny})=\mathrm{Ezt}(\mathrm{Nx}, \mathrm{Ny}-1)$

\section{RESULTS}

The above steps are coded for stratified media. The numerical data generated by the program code were sampled at various time steps from t0 $=10$ to 90 time steps along the computational space and plotted. Figure 1 illustrates the propagation of half -sine pulse introduced at the computation space. In the first case, imagine a pulse to occur at some point within a very low conducting medium with the profile of half sine function at ten time steps i.e. $t 0=10$ as shown in Fig 1a. For the second case imagine the same pulse enters a low conductive medium, thirdly, a pulse propagating into a conductive medium, with conductivity that is relatively higher than the second case and so on. The conductivity of the medium increase with increase in depth as the pulse enters into the medium. 

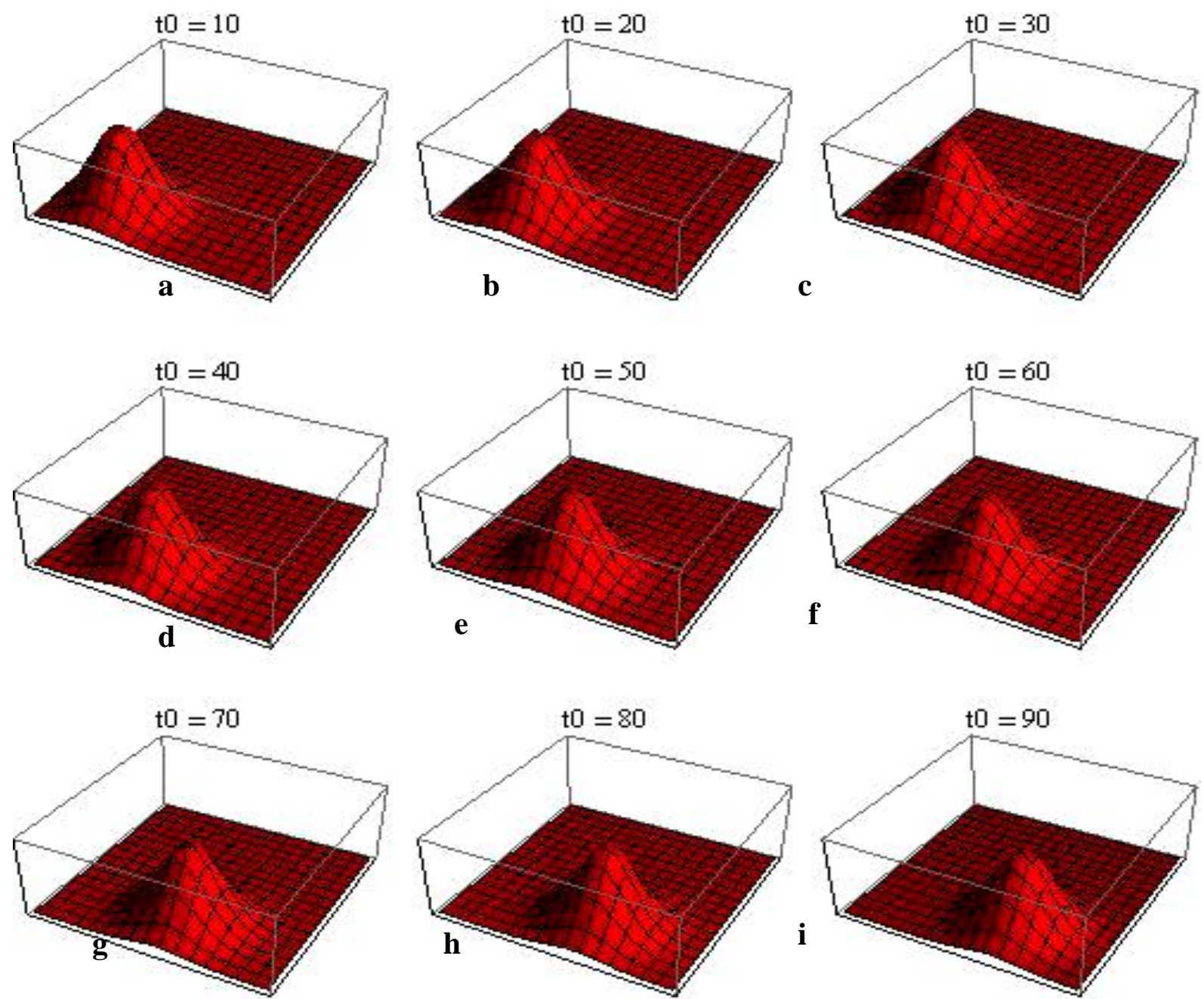

Fig.1: Pulse at different time step

Figure 1a, illustrate the pulse generated at 10 time steps, b, c, d, e, f, g, h and i shows the same pulse at $20,30,40,50,60,70,80$ and 90 time step respectively.

The figures shows the advancement of the pulse into the medium, observed shift in the peak of the amplitude of the pulse when $t 0=10$ and $\mathrm{t} 0=90$. The figures could not show the attenuation of the pulse as the scale is removed to plot more pulse at different time step for transparency. An attempt to show the attenuation is made see figures $2 \mathrm{a}, 2 \mathrm{~b}$ and $2 \mathrm{c}$. 

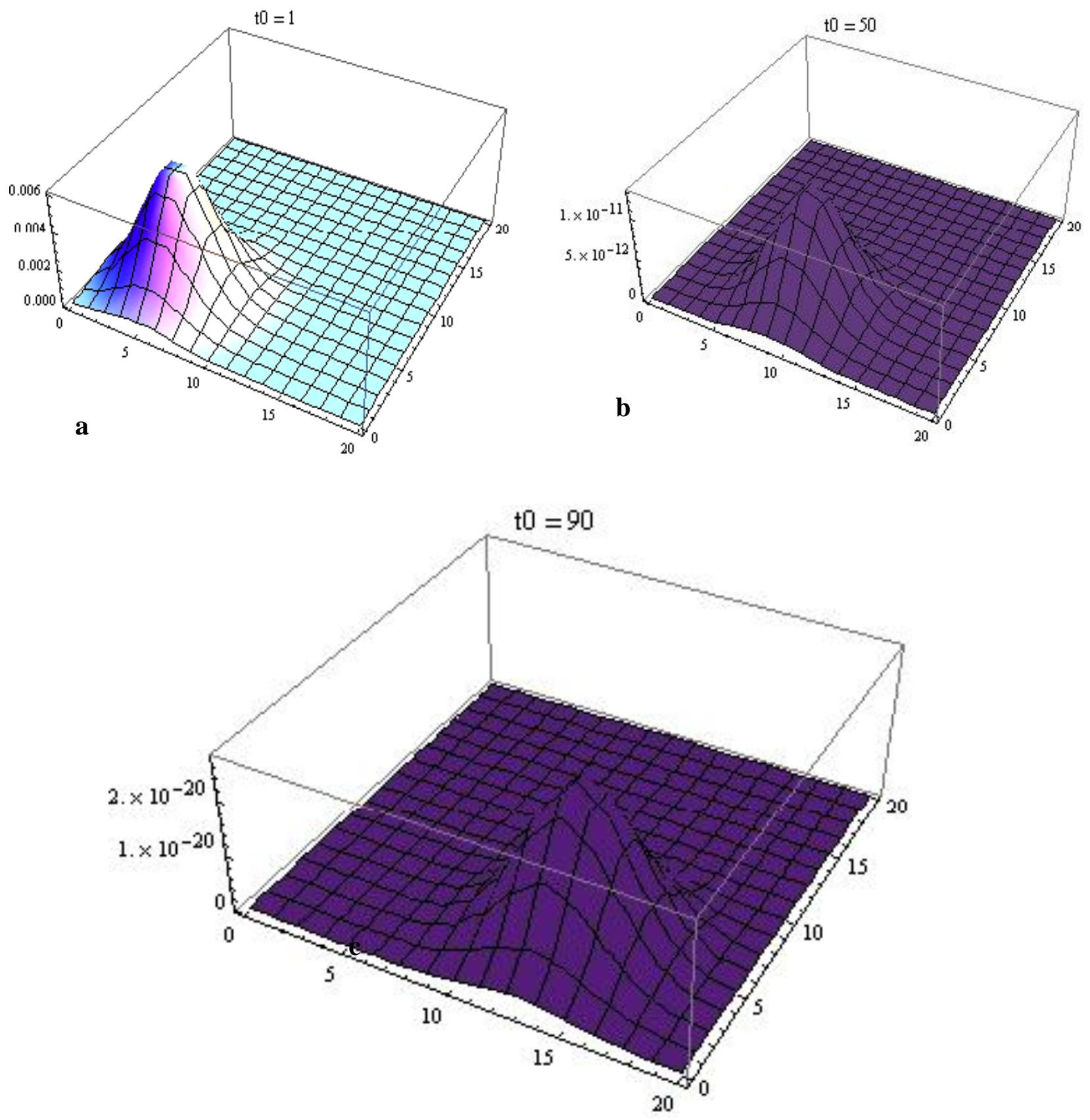

Fig. 2: Advancement of pulse

Fig. 2a shows the entry of the wave into region of very low conductivity at time step 1 , the conductivity is taken to be very small. The attenuation recorded is insignificant as the amplitude of the pulse is 0.006 . At time step 50 in fig. $2 \mathrm{~b}$, the conductivity of the medium is high, the amplitude of the pulse falls sharply from 0.006 to $1 \times 10^{-11}$ representing about $99.99 \%$ 
decrease in amplitude coefficient. This further drastically falls to $2 x 10^{-20}$ for time step 90 as shown in fig 2c which is 2 order magnitudes lower in attenuation of the signal as compared with that in fig $2 \mathrm{~b}$. The attenuation of the signal is accounted by the fact that, wave normally in a medium with finite non-zero conductivity; the wave is being absorbed by the medium as it propagates, because the non-zero conductivity gives rise to an energy loss through joule heat (representative of a dissipative medium). This is similar results presented by [9], modeling borehole radar electromagnetic wave propagation in conductive media. The scattering from the sharp edge of the ore body interferes with the reflected waves and does increase or decrease the amplitude of the received wave depending upon the relative path length. As the conductivity of the host rock increases and, hence, intrinsic attenuation increases, the amplitude of the reflected wave decreases.

\section{CONCLUSION}

FDTD technique is one of the key simulation tools in study of electromagnetic propagation. In this paper, we present a solution of Maxwell's curl relation using forward difference method; codes were generated and subsequently implementation in the mathematica environment version 7.0. The code is applied to investigate propagation of EM waves in stratified medium. It was found through simulation that, increase in conductivity or permeability of the medium contributes, in the same way, to an increase in the attenuation of the signal.

\section{REFERENCE}

Yee, K. S., 1966. "Numerical solution of initial boundary value problems involving Maxwell's equations in isotropic media", IEEE Trans. on Antenna and Propagation, AP-14, (3): 302-307.

Yan, W and Ian W., March 2009. "Introduction to the Segmented Finite-Difference TimeDomain Method" IEEE Transactions on Magnetics, 45, (3): 1364-1367.

Ikata, E., "Electromagnetic waves Basic theory and Computational Technique" 1-133, 270-315.
Hecht, E., 2001. "Optics, 4th ed., Pearson Education”, ISBN 0-8053-8566-5.

Hung, L., 2004. "1D-FDTD using MATLAB" ECEN-6006 numerical methods in photonics project-1,

Vahid, N., Mohammed, S and Mojtaba, D., September 2011. "Analytical and Numerical Calculation of Reflection from a Stratified Structure Backed by a PEMC", IEEE Mediterranean Microwave Symposium (MMS), 134-137, 8-10.

Monorchio, A and Mittra, R., 1998. "Time-domain (FE/FDTD) technique for solving Complex electromagnetic problems", IEEE Microwave and Guided Wave Letters, 8, (2): 93-95, ISSN: 1051-8207, DOI: $10.1109 / 75.658652$,

Demarest, K., Plumb, R and Zhubo, H., 1995. "FDTD modeling of scatters in stratified media" IEEE Transaction on Antennas and Propagation 43, (10): 1164 - 1168, ISSN: $0018-926 \mathrm{X}, \quad \mathrm{DOI}$ 10.1109/8.467657.

Muhkopadhyay, P. K., Wilkinson, A. J., Bennett, T and Inggs, M. R., December 2009. "Modeling borehole radar electromagnetic wave propagation in conductive media by implementation of parallel threedimensional finite-difference time domain technique" Geohorizons, 17-26.

Chen, Y. H and Oristaglio, M. L., 2002. "A modeling study of borehole radar for oilfield applications", Geophysics, 67, (5): 1486-1494.

Chen, Y.-H., Chew, W. C and Oristaglio, M. L., 1997. "Application of perfectly matched layers to the transient modeling of subsurface problems, Geophysics, 62, (6): 17301736.

Wang, D and McMechan, G. A., 2002. "Finitedifference modeling of borehole ground penetrating radar data", Journal of Applied Geophysics, 49, 111-127.

^ Stephen Wolfram: Simple Solutions; The iconoclastic physicist's Mathematica 
software nails complex puzzles, Business Week, October 3, 2005. $\hat{\wedge}$ Wolfram Research Contact Info available on http://www.wolfram.com/company/contac $\mathrm{t} /$ [Accessed on 30/11/2012] 


\section{APPENDIX A}

Glossary of primary variables which appears in the pseudo code

$x 1, x 2, y 1, y 2$ : Coordinate which define the computation boundary planes

$\mathrm{Nx}, \mathrm{Ny}$ : number of subdivisions along $\mathrm{x}$ or $\mathrm{y}$ axis

$\mathrm{N}$ : number of time steps

Delx, Dely: space increment in $\mathrm{x}$ or $\mathrm{y}$ direction

DelT: time increment

Lambd: wave length

Tmax: maximum number of time steps

SF: source function

$\mathrm{Hx}, \mathrm{Hy}$ : Magnetic strength along $\mathrm{x}$ or $\mathrm{y}$ at a point

Ez: electric strength along at a point

CLF: courant factor

Sigma: conductivity of the medium

Vc: velocity of light in free space

Vo: velocity of light in the medium

muo, epo: permeability and permittivity of free space respectively

mur, epr: permeability and permittivity of the medium respectively 